\title{
AGP Related Evaluation of Medical Nutrition Therapy for Diabetes Management (AMEND) - A Real World Observation Study
}

Sanjeev Phatak MD ${ }^{1}$, Banshi Saboo MD, (Ph.D) ${ }^{2}$, Mala Dharamlingam MD DM ${ }^{3}$, Parag Shah DM, DNB ${ }^{4}$, Rajeev Chawla MD ${ }^{5}$, Rajiv Kovil ${ }^{6}$, Sunil M Jain MD, DM7 ${ }^{7}$ Uday Phadke MBBS, MD, DNB ${ }^{8}$, Y Sadasiva Rao MBBS, DD ${ }^{9}$, Reecha Patel M.Pharm ${ }^{10}$ and Nilesh Goswami MBBS, FFM (Fellowship in Family Medicine) ${ }^{11 *}$

${ }^{1}$ Diabetologist and Metabolic Physician, DHL Research Centre, Ahmedabad, India

${ }^{2}$ Chief Diabetologist \& Chairman of Diabetes Care \& Hormone Clinic, Ahmedabad, India

${ }^{3}$ Director BEDRC, Bengaluru, India

${ }^{4}$ Gujarat Endocrine Centre, Ahmedabad, India

${ }^{5}$ Senior Consultant Diabetologist, Director, North Delhi Diabetes Centre, India

${ }^{6}$ Dr. Kovil's Diabetes Care Centre, Mumbai, India

${ }^{7}$ Managing Director at TOTALL Diabetes Hormone Institute, Indore, India

${ }^{8}$ Sahyadri Hospital, Pune, India

${ }^{9}$ Diabetologist, Diabetic care centre, Vijaywada, India

${ }^{10}$ Assistant Manager, Medical Services, Eris Lifesciences, Mumbai, India

${ }^{11}$ Cluster Head Medical Affairs, Eris Lifesciences Ltd., India

*Corresponding author: Nilesh Goswami, Medical Specialist, Medical Affairs, Eris Lifesciences, Ahmedabad, India; E-mail: nilesh.goswami@erislifescience.com

Received: August 04, 2021; Accepted: August 11, 2021; Published: August 18, 2021

\begin{abstract}
Purpose: To evaluate 24 hour glycaemic profile using AGP in patients with type 2 diabetes who are eligible for meal replacement therapy over a period of 14 days.

To assess whether a precise meal replacement plan as an add on to standard of care will make a difference in smoothening out post-prandial peaks and increasing time spent in the desired $(70 \mathrm{mg} / \mathrm{dl}-180 \mathrm{mg} / \mathrm{dl})$ range compared to baseline time in range and post-prandial blood glucose level.

Methods: Patients were mounted with AGP asked to follow the regular diet for 6 days. On the $7^{\text {th }}$ day, based on the AGP report, the most troubled meal was replaced with protein rich, calorie counted, low-carb and fiber enriched meal supplement for the next 6 days. On day 14 , the AGP data were collected.
\end{abstract}

Results: The analysis of full cohort ( $\mathrm{n}=566$ ) showed reduction in eA1c and eAG by $11.9 \%$ (from $7.84 \%$ to $6.90 \%$ ) and $15.10 \%$ (from $178.41 \mathrm{mg} / \mathrm{dL}$ to 151.47 $\mathrm{mg} / \mathrm{dL}$ ), respectively when regular meal diet was compared with the replaced meal diet. The average TIR was improved by $23.56 \%$ (from 41.38 to 51.13 ) in full cohort, post-intervention with replaced meal.

Conclusion: The glycemic profile of patients with type 2 diabetes was improved by meal replacement therapy over period of 14 days.

Keywords: Medical nutrition therapy, Meal replacement, Diabetes, Protein supplement

\section{Introduction}

Diabetes mellitus is a non-communicable, complex and chronic lifestyle-related disorder. It demands continuous medical care with voracious risk-reduction strategies beyond modest glycaemic control. In terms of the sheer prevalence and incidence globally, it may be called a "pandemic", although it is obviously not contagious. Malnutrition, both under- and over-nutrition, is another pandemic and the two co-exist making this a syndemic. Add to this the current COVID-19 pandemic which is more severe in uncontrolled diabetes patients and in those who are overweight or obese.
The recent IDF-2019 report shows that there were $\sim 463$ million adults (20-79 years) living with diabetes; by 2045 this will rise to 700 million [1]. This shows that the incidence of type 2 diabetes mellitus (T2DM) is increasing by leaps and bound across the world. In India, there were $>77$ million people living with diabetes in 2019, which is expected to be 101.5 million by 2030 and 134.2 million by 2045 [2] Moreover, according to a 10-Year Follow-up of the Chennai Urban Rural Epidemiology Study (CURES), among individuals with normal glucose tolerance (NGT), the rate of conversion from prediabetes to diabetes was recorded highest at 18\% [3], indicating that India may overtake China in the near future and become the diabetes capital of the world. 
Since, diabetes is a metabolic disorder, food and lifestyle changes play major role in management of diabetes. Even textbooks and standard guidelines have always mentioned that lifestyle modification needs to be attempted even before metformin, and of late, medical nutrition therapy (MNT) has become a discipline by itself in the management of diabetes.

DiRECT study was the first to provide evidence from a randomised trial of a dietary and lifestyle intervention where type 2 diabetes remissions were the primary outcome [4]. The recent EASD-ADA consensus document released at the EASD meeting in Berlin in October 2018 [5] gives a lot of emphasis on MNT even before metformin.

The Ministry of Health and Family Welfare (MOHFW) has published data that Indians on an average are protein deficient (11\% protein in their diet as against the requisite $15-20 \%$, carbohydrate content in diet is $>65 \%$ as against the needed $55-60 \%$ ) [6].

Many studies have shown that increasing protein intake particularly by whey protein and following portion control with meal replacement plans will benefit diabetes patients by reducing postprandial blood glucose and HbAlc $[4,7,8]$.

Whey protein has essential amino acids that improve insulin secretion as well as sensitivity and when digested, bioactive peptides are formed which have effects on the incretin axis (GLP-1, GIP), they suppress the only orexigenic hormone, Ghrelin, and increase gut anorexigenic hormones such as CCK, NPY and Peptide YY, all of which induce satiety [8-15].

The study was based on the hypothesis that when diabetic patients (uncontrolled with oral anti-diabetic drugs/insulin) were put on protein enriched, low calorie and carbohydrate counted meal replacement plan, will come towards goal HbAlc with reduction in average blood glucose, reduction in estimated Alc (glucose management indicator), and increase in time in range (TIR).

The aim of the study was to carry out a real world evaluation of Medical Nutrition Therapy (MNT) in Patients with Type 2 Diabetes (AMEND - AGP related evaluation of MEdical Nutrition therapy for Diabetes management).

The primary objective was to evaluate 24 hour glycaemic profile using AGP in patients with type 2 diabetes who are eligible for meal replacement therapy over a period of 14 days. The secondary objective was to assess whether a precise meal replacement plan as an add on to standard of care will make a difference in showing how it smoothens out post-prandial peaks and increases time spent in the desired (70 $\mathrm{mg} / \mathrm{dl}-180 \mathrm{mg} / \mathrm{dl}$ ) range compared to baseline time in range and postprandial blood glucose level.

\section{Subjects}

The study enrolled patients with diabetes who were 18 years old male or female and preferably overweight (BMI $\geq 24$ or metabolically obese normal weight). The newly diagnosed patients with type 2 diabetes or patients already on treatment were also eligible. Participating participants were physically and mentally able to give valid informed written consent form.
The exclusion criteria for subjects were as below. 1) HbA1c $>10 \%$, BMI $>38$, EGFR $<45$ (any one of this will not allow participants to be eligible for the study). 2) Participants suffering from any acute illness, CVD, renal complications, bowel disorders or eating disorders like anorexia or bulimia. 3) Participants who are consuming antipsychotics, steroids or GLP-1 analogues.4) Participant whose diabetes was diagnosed $>10$ years ago or who has undergone bariatric surgery. 5) Participants who are unable or unwilling to take prescribed precise meal replacement plan and/or not willing to sign the informed consent form. 6) Pregnant or lactating women, cancer patients. 7) Participants who are already on some diet plan or following fast during the study or consuming protein supplements or consuming alcohol on daily basis or are allergic to the ingredients of the Simetri meals or Prototal. 8) Participants are not allowed to make any significant change in the daily routine or physical activities.

\section{Materials and Methods}

In a real world setting, when the patient with type 2 diabetes visited the doctor, after taking the consent, they were enrolled in the routine evaluation. After completion of all screening assessments (i.e., demographic, anthropometric and clinical data) and obtaining signed informed consent forms, eligible patients were mounted with flash glucose monitoring system (Free Style Libre from Abbott for 14 day) to check their Ambulatory Glucose Profile (AGP). Patients were given a food log sheet and asked to fill the same for the next 14 days.

The first 6 days were considered as a baseline phase where patients continued on their regular food without any major changes. The $7^{\text {th }}$ day was the meal replacement phase when the patient visited the doctor and on the basis of the AGP report, investigator replaced - the most troubled meal (the one which was associated with the most postprandial glycaemic excursions) - regular food with a customized meal replacement diet which included Simetri meals (From Eris Lifesciences - a calorie counted, protein enriched, ready to eat meal - details in supplement material) and or ProTotal (From Eris Lifesciences - a whey protein supplement - $14 \mathrm{gm}$ whey protein, $6.6 \mathrm{gm}$ of fibre with other macro and micronutrients). The most troubled meal was identified as the maximum post-prandial peak seen in the AGP report on day $7^{\text {th }}$. Patients were asked to consume Simetri and/or ProTotal for the next 7 days (replaced meal phase) without any change in their medications. Here, the same patient has served as the control for himself which could be more accurate than a parallel group design (inter-individual variation). Throughout the study period (14 days) the investigator did not make any change in the pharmacological treatments.

AGP device was mounted on subject by investigator on day 1 of the study. The AGP device has been carried by the subject till day 14 of the study. The data was extracted from the device by investigator in a timely manner, i.e., on day 7 and day 14 .

Given the observational nature of the study, no sample size estimation was done. Statistical analysis was carried out as and when required. The level of significance was 0.05 . $P$ values of less than 0.05 were considered as a statistically significant difference. 


\section{Ethical Conduct of the Study}

The clinical study protocol (Dated 01/02/2019), informed consent form, subject diary (Version No. 00,Dated 12 Apr 2019) and all other relevant study documentation were reviewed and approved by the responsible ethics committee.

The study commenced only after a written approval was obtained from the ethics committee. The study was conducted in accordance with the protocol, International Council for Harmonisation (ICH) (Step 5) 'Guidance on Good Clinical Practice' (E6) and 'Declaration of Helsinki'. All associates assisting in the conduct of study were informed regarding their obligations.

\section{Results}

There were total 660 patients enrolled and out of them 566 patients were involved in the final analysis. We have divided patients in various groups for the purpose of better analysis.

Total 94 (14.24\%) non-adhered patients were excluded from efficacy analysis. Non-adherent patients were defined as the one who had consumed the provided replaced meal (Simetri and Prototal) for $<4$ days. The adherence to diet was observed in $85.76 \%$ of total 660 patients.

In all patients age was found to be in a range of 20-86 years with a mean of $53.49 \pm 11.80$ years, mean weight was $73.77 \pm 14.33 \mathrm{~kg}$, and mean BMI was $27.81 \pm 5.52 \mathrm{~kg} / \mathrm{m}^{2}$. The mean HbA1c was $9.10 \pm$ $1.66 \%$.

\section{a. Full cohort}

The analysis of full cohort $(\mathrm{n}=566)$ showed reduction in average eA1c (Figure 1) from 7.84 on regular diet to 6.90 on replaced diet, indicating an eA1c (estimated A1c - it was estimated because the total duration is only 14 days) reduction by $11.99 \%, \mathrm{p}<0.001$. Similarly, estimated average glucose (eAG) on regular diet was $178.41 \mathrm{mg} / \mathrm{dL}$ which reduced to an avg. of $151.47 \mathrm{mg} / \mathrm{dL}$ after following replace diet, indicating reduction by $15.10 \%, \mathrm{p}<0.001$. The average TIR on regular diet was 41.38 which improved to an avg. of 51.13 after following replace diet, indicating an improvement by $23.56 \%$ (Figure 2). This improvement showed statistical significant change $(p<0.001)$.

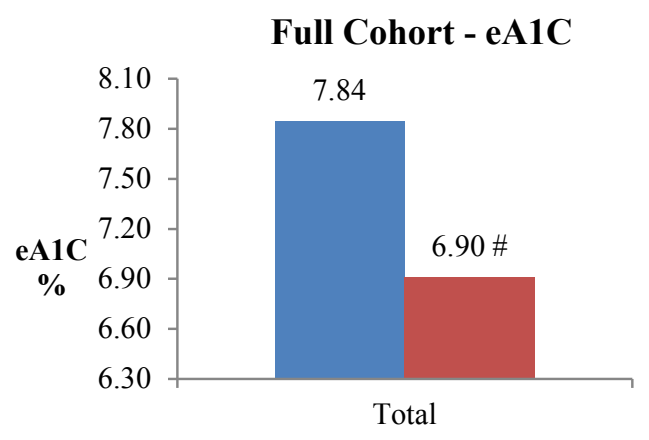

Average of eA1C-REG DIET (\%)

- Average of eA1C-REP DIET (\%)

$\#$ P value $<0.001$

Figure 1: Full cohort analysis of eAlc.

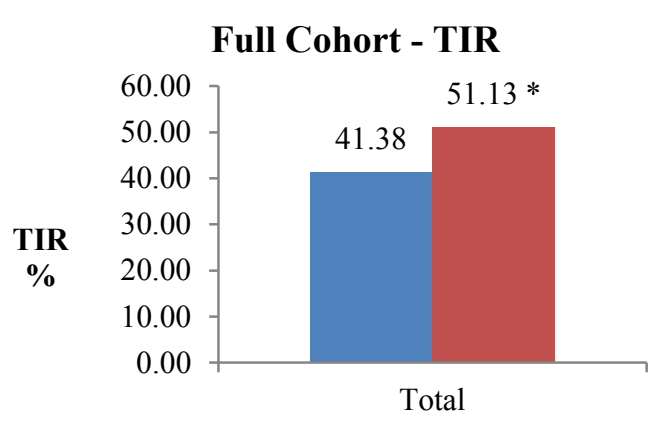

- Average of AVG-TIR REG DIET (\%)

- Average of AVG-TIR REP DIET (\%)

${ }^{*} \mathrm{P}$ value $<0.001$

Figure 2: Full cohort analysis of TIR.

\section{b. Sub-group analysis}

The age group analysis (Table 1 ) showed that patients $>60$ years had better improvement in eAG and eAlc $-16.73 \%$ and $13.10 \%$ respectively when compared to age group of $<40$ years and $40-60$ years ( $14.35 \%$ and $11.46 \%$ vs. $14.47 \%$ and $11.54 \%$, respectively)

The sub-group analysis for various BMI indicated that group of patients with BMI between 25 to $29.9 \mathrm{~kg} / \mathrm{m}^{2}$ had $15.02 \%$ and $11.88 \%$ reduction in eAg and eA1c when replaced meal was consumed whereas patients with BMI $>30 \mathrm{~kg} / \mathrm{m}^{2}$ had comparatively less improvement in eAG (11.09\%) and eA1c (8.79\%) on replaced meal where $\mathrm{p}<0.001$, as shown in Table 1 . However, improvement in TIR was slightly greater in patients with higher BMI (23.19\% vs. $21.58 \%)$.

Analysis was also carried out based on the duration of diabetes. Patients with diabetes duration between 1 to 6 years and $>6$ years showed better improvement than patients with diabetes duration $<1$ year. Improvement in eAG, eA1C and TIR was $15.54 \%, 12.33 \%$ and $24.46 \%$ respectively in patients with diabetes duration 1 to 6 years, while in patients with $>6$ years the improvement was by $15.46 \%$, $12.36 \%$ and $25.82 \%$ respectively (Table 1 ).

When patients were divided based on the avg. baseline HbAlc < $7.5 \%$ and $>7.5 \%$, the result showed that patients whose avg. baseline $\mathrm{HbAlc}$ was $<7.5 \%$ there was decrease in eAG from $144.25 \mathrm{mg} / \mathrm{dL}$ on regular diet to $117.27 \mathrm{mg} / \mathrm{dL}$ on replaced diet indicating a decrease by $18.70 \%$. In the same group, eA1c decreased by $14.14 \%, \mathrm{p}<0.001$. On the other hand, patients' whose avg. baseline HbAlc was $>7.5 \%$ also showed significant improvement with $\mathrm{p}<0.001$ in eAG and eA1c ( $14.85 \%$ and $11.82 \%$ respectively).

As it was a real world study, a few of the patients were consuming either Simetri or ProTotoal and most of them were consuming both Simetri and ProTotal. There was also a small group of patients who were consuming more than two replaced diet in a day. The analysis was carried out to observe the effect of single meal replacement vs. two meal replacements in a day. As shown in the Table 1, patients consuming two replaced diets in a day showed better results in terms of all the evaluated parameters. 
Table 1: eAG, elc and TIR values of various sub-group analysis.

\begin{tabular}{|c|c|c|c|c|c|c|c|c|}
\hline \multirow{2}{*}{ Subgroup } & \multirow{2}{*}{ No. of subjects } & \multicolumn{2}{|c|}{ eAG (mg/dL) } & \multicolumn{2}{|c|}{ TIR (\%) } & \multicolumn{2}{|c|}{ eAlc (\%) } & \multirow{2}{*}{ p value } \\
\hline & & Regular diet & Replaced diet & Regular diet & Replaced diet & Regular diet & Replaced diet & \\
\hline $\mathrm{BMI}<24.9 \mathrm{~kg} / \mathrm{m}^{2}$ & 161 & 185.62 & 151.93 & 37.79 & 48.09 & 8.09 & 6.92 & $<0.001$ \\
\hline $\mathrm{BMI}=25-29.9 \mathrm{~kg} / \mathrm{m}^{2}$ & 242 & 173.13 & 147.12 & 43.42 & 52.79 & 7.66 & 6.75 & $<0.001$ \\
\hline $\mathrm{BMI}>29.9 \mathrm{~kg} / \mathrm{m}^{2}$ & 151 & 175.46 & 156.01 & 43.25 & 53.28 & 7.74 & 7.06 & $<0.001$ \\
\hline$<40$ years & 71 & 181.13 & 155.13 & 44.37 & 53.81 & 7.94 & 7.03 & $<0.001$ \\
\hline $40-60$ years & 326 & 181.99 & 155.65 & 39.41 & 48.84 & 7.97 & 7.05 & $<0.001$ \\
\hline$>60$ years & 169 & 170.36 & 141.86 & 43.94 & 54.41 & 7.56 & 6.57 & $<0.001$ \\
\hline $\mathrm{DD}<1$ year & 28 & 128.99 & 113.63 & 64.43 & 73.39 & 6.12 & 5.59 & $<0.001$ \\
\hline DD 1-6 years & 179 & 179.19 & 151.34 & 42.32 & 52.67 & 7.87 & 6.9 & $<0.001$ \\
\hline $\mathrm{DD}>6$ years & 343 & 183.08 & 154.78 & 38.62 & 48.59 & 8.01 & 7.02 & $<0.001$ \\
\hline eAlc reg diet $<7.5 \%$ & 295 & 133.97 & 118.46 & 62.06 & 66.27 & 6.3 & 5.75 & $<0.001$ \\
\hline eAlc reg diet $>7.5 \%$ & 271 & 226.78 & 187.41 & 18.88 & 34.65 & 9.53 & 8.16 & $<0.001$ \\
\hline One meal replacement & 170 & 166 & 144.76 & 45.48 & 54.21 & 7.41 & 6.67 & $<0.001$ \\
\hline Two meal replacement & 392 & 183.82 & 154.28 & 39.74 & 49.96 & 8.03 & 7 & $<0.001$ \\
\hline
\end{tabular}

DD: Diabetes Duration.

Table 2: Evaluation parameters when single replaced meal is ProTotal.

\begin{tabular}{|l|c|}
\hline Parameters & One meal replacement with ProTotal $(\mathbf{N}=64)$ \\
\hline eAG difference & $16.68 \mathrm{mg} / \mathrm{dL}$ \\
\hline$\%$ Reduction in eAG & $10.23 \%$ \\
\hline eAlc difference & 0.17 \\
\hline$\%$ Reduction in eAlc & $2.33 \%$ \\
\hline TIR improvement & $18.82 \%$ \\
\hline P value & $<0.001$ \\
\hline
\end{tabular}

We also evaluated patients whose one replaced meal was ProTotal to see the benefits of whey protein on eAG, eA1c and TIR. The results are presented in Table 2 .

\section{Discussion}

From the latest figures of IDF we know that India ranks second highest among people with diabetes after China [2]. The worrisome part is that as per the National Results of the SITE (Screening India's Twin Epidemic) study, of the diabetic population already aware of their condition, almost two-of-every three (70\%) had 'uncontrolled' diabetes (Hbalc levels $>7 \%$ ) [16].

This figure raises a question that in spite of availability of a vast range of pharmacological treatments, why does a patient has uncontrolled diabetes and what is the missing link? The answer can be obtained from the STARCH study [17] and PRODIGY survey [18] which showed that protein intake in India is very less and there hardly any difference exists between diets of a non-diabetic patient to that of a diabetic patient. It was observed that around 9 out of 10 consumers had a diet deficient in proteins. This was regardless of the gender and the socio-economic group [18]. Even the MoHFW (Ministry Of Health and Family Welfare) stated that Indians on an average are protein deficient (11\% protein in their diet as against the requisite 1520\%) [5].
As diabetes is a metabolic and lifestyle related disease, controlling patients' diet is one of the key success factors. In fact, lifestyle modification including MNT should be started even before metformin. Unfortunately by the time patients get diagnosed for diabetes, they have already lost $50-80 \%$ beta cell function [19]. Therefore, in this study, we asked patient to replace one or two of their meals with the specially designed, protein-enriched, carbohydrate-counted meal, without changing any pharmacological treatment.

The study enrolled a total of 660 patients. Based on the AGP report at the end of first 6 days, patients were asked to replace the most troubled meal with either Simetri or ProTotal or both for the next 7-8 days. Out of these 660 patients, 94 patients did not follow the study protocol and consumed replaced diet only for a period of $<4$ days. These $14.24 \%$ patients were not included in the analysis. This showed that rate of adherence to diet change was $85.76 \%$. As diet is the hardest thing to change, the significant adherence to given product suggested that the palatability and ease of consumption for Simetri and ProTotal is very high. As it was a real world observation study, there was no control over patients' lifestyle (food choice, exercise pattern, sleeping pattern etc.). The only change was replacing one or two meal with either ProTotal or Simetri or both and there was no strict control over what the patients consume in the entire day.

In the full cohort, eA1c was reduced by $0.94 \%$ (from $7.84 \%$ to $6.90 \%$; \% improvement $11.99 \%, \mathrm{p}<0.001$ ). The results were similar to that obtained with GLP-1 agonist in AMIGO study where exenatide $10 \mu \mathrm{g}$ resulted in a mean $\mathrm{HbAlc}$ reduction of $-0.8 \%$ to $-0.9 \%$ [20]. As whey protein also improves GLP-1 secretion, it can mimic the action of GLP-1 agonist [8]. This indicates that, even a small change in a diet for only a short duration of 7 days has very positive impact on eAlc and if patient continues following the suggested meal replacement plan it will serve like a pharmacological therapy. Even the UKPDS study has shown that intensive glucose control can significantly reduce any diabetes related end-point [21]. 
As per the recommendations from the international consensus on time in range (TIR), adults with type 1 or type 2 diabetes should have $>70 \%$ of TIR. In our study, when patients were on regular diet and average TIR was only $41.38 \%$ which increased to $51.13 \%$ after consuming replaced diet. This indicates that, if patients continue following the replaced diet there will be much improvement in TIR. Though in the current study, patient consumed replaced diet only for 7 days, still an increase in TIR is really significant (relative \% improvement of $23.56 \%, \mathrm{p}<0.0001$ ).

The replace diet not only improved TIR but also decreased the eAG from $178.41 \mathrm{mg} / \mathrm{dL}$ to $151.47 \mathrm{mg} / \mathrm{dL}, \mathrm{p}<0.001$ in a full cohort.

The similar result were obtained when different sub-group analysis were carried out, i.e., based on the BMI, baseline HbAlc and the number of replaced meals.

There was no difference seen in male and female patients with reference to improvement. It means the replaced diet causes the similar effect on both the gender. The meal replacement period was only for short duration of days hence change in weight was not included in the evaluation parameter and measuring $\mathrm{HbAlc}$ was also not possible.

The results from DiRECT and DiRECT 2 studies support that total diet replacement for initial 12-20 weeks followed by gradual food reintroduction and then support for weight loss maintenance has helped type 2 diabetes patients to achieve remission and sustained it at 24 months for more than a third of people with type 2 diabetes [22].

The AMEND study results were also in line with these studies. Although the study duration was very short and hence measuring a change in body weight was not feasible, the eAG, eA1c and TIR results suggested that if the treatment was continued for longer duration, there are chances that blood glucose and $\mathrm{HbAlc}$ will be in the controlled range.

AMEND study results were also supported by David King (2018) study which concluded that whey protein before meals improved postprandial glycemia, stimulated insulin release, and increased satiety in men with type 2 diabetes [23].

The protein quantity and quality of foods are contributing factors to their effects on glucose control, but foods are much more complex than a single nutrient, or even the sum of their individual nutrients. Many dietary factors, nutritive and/or bioactive, mediate the relationship between food intake and health. Lastly, in order to optimize protein intake for glucose regulation, the amount, source, and type of food product or supplement should be personalized to match to the individual's lifestyle, medications, gluco-regulatory abilities, and disease status.

\section{Conclusion}

This AMEND study showed that the efficacy of replaced diet for calorie restriction remains a significant in humans. Although this data was only for 14 days, the study can give significant and detailed data on effect of replaced diet in patients with diabetes if therapy continues for longer period of time. Improving diet of a patient will not only help them in better management of a disease but will also be cost-effective for the country [24].
Therefore, it can be concluded from the present study that, replacing a diet with protein enriched, calorie counted meal does not only improve the blood glucose level and TIR but can also decrease eA1c significantly.

\section{Acknowledgement}

We would like to thank all physicians who have helped in the study. We would also like to extend our gratitude to CRO - Ethicare for their help in data analysis.

\section{Declaration}

\section{Funding}

Eris Lifesciences Pvt. Ltd

\section{Conflicts of Interest/Competing Interests}

Not applicable

\section{Ethics approval}

Approved. Aastha Ethics Committee has given the approval.

\section{Informed Consent}

Informed consent was obtained from all individual participants included in the study.

\section{Consent for publication}

Each author has given their consent for publication.

\section{Availability of data and material}

Not applicable.

\section{Code availability}

Not applicable.

\section{References}

1. IDF Diabetes Facts and Figures. IDF Diabetes Atlas Ninth edition 2019. https://www. idf.org/aboutdiabetes/what-is-diabetes/facts-figures.html. Accessed 17 June 2020.

2. IDF Diabetes Atlas 9th edition, 2019. https://diabetesatlas.org/en/sections/ demographic-and-geographic-outline.html. Accessed 17 June 2020.

3. Ranjit A, Coimbatore R, Mohan D, Rajendra P, Vasudevan S, Haridas N, et al (2015) Incidence of diabetes and prediabetes and predictors of progression among asian indians: 10-year follow-up of the Chennai Urban Rural Epidemiology Study (CURES). Diabetes Care 38(8): 1441-8. [crossref]

4. Michael L, Wilma L, Alison B, Naomi B, George T, Lousie M, et al. (2018) Primary care-led weight management for remission of type 2 diabetes (DiRECT): an openlabel, cluster-randomised trial. Lancet 391: 541-551. [crossref]

5. Melanie Davies, David D, Judith F, Walter K, Chantal M, Geltrude M, et al. (2018) Management of hyperglycaemia in type 2 diabetes. A consensus report by the American Diabetes Association (ADA) and the European Association for the Study of Diabetes (EASD), Diabetologia, Springer Publications, EASD and ADA. Diabetologia 61: 2461-2498. [crossref]

6. Press Information Bureau Government of India Ministry of Health and Family Welfare, Nutritional Level. 13-March-2015.

7. Bhatt A, Choudhari K, Mahajan R, Sayyad G, Pratyush D, Hasan I, et al. (2017) Effect of a low-calorie diet on restoration of normoglycemia in obese subjects with type 2 diabetes. Indian J Endocr Metab 21: 776-80. [crossref]

8. Linda M, Tongzhi W, Michael H, Christopher R (2015) Whey protein: The "whey" forward for treatment of type 2 diabetes? World J Diabetes 6(14): 1274-84 
9. Hall L, Millward J, Long J, Morgan M (2003) Casein and whey exert different effects on plasma amino acid profiles, gastrointestinal hormone secretion and appetite. $\mathrm{Br} J$ Nutr 89: 239-48. [crossref]

10. Nilsson M, Holst J, Björck M (2007) Metabolic effects of amino acid mixtures and whey protein in healthy subjects: studies using glucose-equivalent drinks. Am J Clin Nutr 85(4): 996-1004. [crossref]

11. Salehi A, Gunnerud U, Muhammed J, Ostman E, Holst J, Björck I, et al. (2012) The insulinogenic effect of whey protein is partially mediated by a direct effect of amino acids and GIP on $\beta$-cells. Nutr Metab 9: 48. [crossref]

12. Kevin C, Gonca P (2016) Emerging evidence for the importance of dietary protein source on glucoregulatory markers and type 2 diabetes: different effects of diary, meat, fish, egg and plant protein foods. Nutrients 8: 446. [crossref]

13. Nicole K, Melinda C, Gayle S, Christopher R (2014) Effect of dietary prebiotic supplementation on advance glycation, insulin resistance and inflammatory biomarkers in adults with pre-diabetes: a study protocol for a double-blind placebo-controlled randomised crossover clinical trial. BMC Endocrine Disorders 14: 55. [crossref]

14. Cristina T, Harriët S, Timothy D, John C (2017) The microbiota-gut-brain axis in obesity. Lancet Gastroenterol Hepatol 2(10): 747-56. [crossref]

15. Blaak E, Antoine M, Benton D, Björck I, Bozzetto L, Brouns F, et al. (2012) Impact of Postprandial Glycaemia on Health and Prevention of Disease. Obesity Reviews 13(10): 923-84. [crossref]

16. National results of nearly 16,000 patients surveyed for Sanofi's SITE study show that $70 \%$ of the known diabetic population have uncontrolled diabetes. Media release by Sanofi India on 7 November 2011.
17. Shashank J, Anil B, Sarita B, Subodh B, Mala D, Shachin G, et al. (2014) Results from a dietary survey in an Indian T2DM population: a STARCH study. BMJ Open 4(10): e005138. [crossref]

18. Manish Mahajan (2015) Protein Consumption in Diet of Adult Indians: A General Consumer Survey (PRODIGY). Indian Medical Gazette 149-50.

19. Baptist Gallwitz (2006) The Fate of Beta-cells in Type 2 Diabetes and the Possible Role of Pharmacological Interventions. Rev Diabetic Stud 3(4): 208-16. [crossref]

20. Dilip S, Suril V, Shivani V, Kiran K, Vinod T (2018) Recent updates on GLP-1 agonists: Current advancements \& challenges. Biomed Pharmacother 108: 952-962. [crossref]

21. Paromita K, Ian P, Richard D (1999) The UK Prospective Diabetes Study (UKPDS): clinical and therapeutic implications for type 2 diabetes. Br J Clin Pharmacol 48(5): 643-8. [crossref]

22. Michael L, Wilma L, Alison B, Naomi B, George T, Louise M, et al. (2019) Durability of a primary care-led weight-management intervention for remission of type 2 diabetes: 2-year results of the DiRECT open-label, cluster-randomised trial. Lancet Diabetes Endocrinol 7(5): 344-55. [crossref]

23. David K, Mark W, Matthew C, Leigh B, Emma S, Daniel W (2018) A small dose of whey protein co-ingested with mixed-macronutrient breakfast and lunch meals improves postprandial glycemia and suppresses appetite in men with type 2 diabetes: a randomized controlled trial. Am J Clin Nutr 107(4): 550-7. [crossref]

24. Lee Y, Mozaffarian D, Sy S, Huang Y, Liu J, Wilde PE, et al. (2019) Cost-effectiveness of financial incentives for improving diet and health through Medicare and Medicaid: A microsimulation study. PLoS Med 16(3): e1002761. [crossref] 